\title{
Expression of Biomarkers (CD44, COX-2, TWIST, VEGF-C and LOX) in Esophageal Cancer: Implication for Prophylactic Radiation Field
}

\author{
Yasuhito Hagiwara $^{1,}$ *, Kenji Nemoto ${ }^{1}$, Ibuki Ota ${ }^{2}$, Masayoshi Yamada ${ }^{1}$, Osamu Hachiya ${ }^{3}$, \\ Mitsunori Yamakawa ${ }^{4}$ \\ ${ }^{1}$ Department of Radiation Oncology, Yamagata University Faculty of Medicine, Yamagata, Japan \\ ${ }^{2}$ Department of Radiation Therapy, Iwate Prefectural Central Hospital, Iwate, Japan \\ ${ }^{3}$ Department of Gastroenterological, General, Breast ant Thyroid Surgery, Yamagata University Faculty of Medicine, Yamagata, Japan \\ ${ }^{4}$ Department of Pathological Diagnostics, Yamagata University Faculty of Medicine, Yamagata, Japan
}

\section{Email address:}

hagiwara.yasuhito@gmail.com (Y. Hagiwara), knemoto@ymail.plala.or.jp (K. Nemoto), o.ibuki.yamagata@gmail.com (I. Ota), msysh111@gmail.com (M. Yamada), ohachiya@med.id.yamagata-u.ac.jp (O. Hachiya),

myamakaw@med.id.yamagata-u.ac.jp (M. Yamakawa)

*Corresponding author

\section{To cite this article:}

Yasuhito Hagiwara, Kenji Nemoto, Ibuki Ota, Masayoshi Yamada, Osamu Hachiya, Mitsunori Yamakawa. Expression of Biomarkers (CD44, COX-2, TWIST, VEGF-C and LOX) in Esophageal Cancer: Implication for Prophylactic Radiation Field. International Journal of Clinical Oncology and Cancer Research. Vol. 6, No. 1, 2021, pp. 10-16. doi: 10.11648/j.ijcocr.20210601.12

Received: December 29, 2020; Accepted: January 8, 2021; Published: January 22, 2021

\begin{abstract}
Background and objective: If it becomes possible to reduce the prophylactic radiation field by using a personalized radiation field, radiation therapy of esophageal cancer may become safer. In this study, we examined the relationship between expression levels of biomarkers (CD44, COX-2, TWIST, VEGF-C and LOX) and lymph node metastasis and distant metastasis. Materials and methods: We performed retrospective analysis of 14 patients who underwent radical surgery for esophageal cancer between 2009 and 2011 in Yamagata University Hospital. Stain index was used for assessment of biomarker expression. The relationships of stain index with regional recurrence and distant metastasis were evaluated. Results: All patients had squamous cell carcinoma. The median follow-up duration was 38 months. Distant failure was significantly related to low COX-2 expression in the deep part of the tumor (stain index $<2.8 ; \mathrm{p}=0.0479$ ). Pathological node-positive or regional recurrence was significantly related to low VEGF-C expression (stain index $<0.3 ; \mathrm{p}=0.0418$ ). Patients with low CD44 expression in the surface of the tumor tended to have better reginal control (stain index $<0.3 ; \mathrm{p}=0.0902$ ). Conclusions: COX-2, VEGF-C, and CD44 expression levels might have potential to enable omission of the prophylactic radiation field. Further analysis using biopsy samples is required for application to radical radiotherapy.
\end{abstract}

Keywords: Esophageal Cancer, CD44 Antigen, Cyclooxygenase 2, Vascular Endothelial Growth Factor C, Lymphatic Metastasis

\section{Introduction}

The outcome of radiation therapy for esophageal cancer has been improved by the development of chemoradiotherapy and by advances in diagnostic imaging using endoscopy, contrast-enhanced computed tomography (CT), magnetic resonance imaging (MRI), and positron emission tomography with 2-deoxy-2-[fluorine-18]fluoro-D-glucose (18F-FDG-PET)
[1-5]. Standardization of treatment regarding dose fractionation of radiotherapy and key drugs for chemoradiotherapy has been achieved. The standard key drugs for chemoradiotherapy are 5-fluorouracil and cisplatin (FP), though there are some difference in the dose and administration interval [6-8]. The standard dose fractionation in Europe and America is 50.4 Gy in 28 fractions [6]. And the standard dose fractionation in Japan is $60 \mathrm{~Gy}$ in 30 fractions [7].

On the other hand, there has not been sufficient 
investigation regarding the prophylactic lymph node irradiation field. Reported incidences of lymph node metastasis are $14-21 \%$ in $\mathrm{T} 1 \mathrm{~b}$ cases and $38-60 \%$ in $\mathrm{T} 2$ cases [9-11]. Furthermore, the pattern of lymph node metastases based on primary site and tumor stage has been reported [12]. Therefore, a prophylactic radiation field has been considered as would be necessary in $\geq \mathrm{T} 1 \mathrm{~b}$ cases in esophageal cancer radiation therapy. Collard et al. [9] showed that $13 \%$ of $\mathrm{T} 1$ patients, $37 \%$ of $\mathrm{T} 2$ patients, and $68 \%$ of $\mathrm{T} 3$ patients have metastatic lymph nodes. However, conversely, this means that about $87 \%$ of $\mathrm{T} 1$ patients, $63 \%$ of T2 patients, and $32 \%$ of T3 patients do not need prophylactic radiation in the lymph node region.

In esophageal cancer radiotherapy, a large irradiation field including a prophylactic radiation field is a risk for lung and heart toxicities [13, 14]. Morota et al. [14] showed that the two-year cumulative incidence of late cardiopulmonary toxicities of $\geq$ grade 3 for patient $\geq 75$ years old was $29 \%$. Determination of the radiation field in cases of esophageal cancer has been based on the primary site and tumor, nodes, and metastases (TNM) staging. This information has been obtained by endoscopy, pathological examination, and diagnostic imaging techniques such as contrast-enhanced CT, MRI, and 18F-FDG-PET. If criteria for expression levels of biomarkers could be added to the risk assessment of lymph node metastasis and distant metastasis, individualization of the radiation field might be possible.

The aim of this study was to find biomarkers that might enable determination of the risk of regional recurrence and distant metastasis. It is uncertain whether prognosis of patients with a high risk for regional recurrence can be improved by using a large prophylactic radiation field. However, prognosis of patients with a low risk for regional recurrence might not be worsened by omitting a large prophylactic radiation field, and omission of a large prophylactic radiation field in such patients might have a positive effect by reducing the toxicities of treatment. In this study, we investigated the relationship between expression of biomarkers and lymph node metastasis. And which biomarker could be candidate for omission of prophylactic radiation fields. Cluster of differentiation 44 (CD44), cyclooxygenase-2 (COX-2), vascular endothelial growth factor C (VEGF-C), TWIST, and lysyl oxidase (LOX) were chosen as biomarkers because these markers have been reported to be related to lymph node metastasis and prognosis in patients with esophageal cancer, non-small cell lung cancer, head and neck cancer, gastric cancer and colorectal cancer [15-20].

\section{Materials and Methods}

\subsection{Inclusion and Exclusion Criteria}

This study was a single-center, retrospective study of 14 patients with primary esophageal cancer who underwent radical surgery between January 2009 and November 2011 at Yamagata University Hospital. Patients provided informed consent authorizing the use of their personal information for research purposes. This study was approved by the appropriate institutional review board (H23-75, Yamagata University Hospital) and was carried out in accordance with the Declaration of Helsinki. The inclusion criteria were as follows: 1) histologically confirmed esophageal cancer, 2) treated with definitive intent, 3) Eastern Cooperative Oncology Group (ECOG) performance status of 0 or 1, and 4) pathological specimens being available. The following patients were excluded: 1) patients who received preoperative chemoradiotherapy, 2) patients who received postoperative radiotherapy, and 3) patients who could not receive complete resection. Preoperative chemoradiotherapy has relative high rates of pathological complete response (26\%) and near pathological complete response (17\%), but preoperative chemotherapy has a low pathological complete response rate $(2.5 \%)[21,22]$. Patients who received preoperative chemoradiotherapy and one patient with incomplete excision were excluded.

\subsection{Immunostaining}

Specimens were fixed in 10\% formalin. Tumor samples were then embedded in paraffin and 4-microm-thick sections were cut. Paraffin sections were used for hematoxylin and eosin staining and for immunohistochemistry. The following antibodies were used for immunohistochemistry: anti-human mouse monoclonal antibody to CD44 (AM00649PU-N, Acris Antibodies; Rockville, United States), anti-human mouse monoclonal antibody to COX-2 (N1606, Dako; Glostrup, Denmark), anti-human rabbit monoclonal antibody to TWIST (sc-15393, Santa Cruz; Texas, United States), anti-human mouse monoclonal antibody to VEGF-C (BS-1586R, Bioss; Massachusetts, United States) and anti-human rabbit polyclonal antibody to LOX (NB110-41568, Novus Biologicals; Colorado, United States). For antigen retrieval, deparaffinized sections were treated with an antigen activation solution $(\mathrm{pH} 9.0$, Nichirei; Tokyo, Japan) in an autoclave for 20 minutes at $120^{\circ} \mathrm{C}$ $(400 \mathrm{~W})$. Endogenous peroxidase activity was blocked by immersion in hydrogen peroxide. Sections were incubated with a primary antibody at $4^{\circ} \mathrm{C}$ overnight. After washing, a mixture of biotinylated goat anti-mouse immunoglobulins and goat anti-rabbit immunoglobulins (Dako; Glostrup, Denmark) was added and incubated for 2 hours at room temperature. After washing, streptavidin-horseradish peroxidase complex (Dako; Glostrup, Denmark) was added and incubated for 30 minutes at room temperature. A positive reaction was visualized with 3,3'-diaminobenzidine (Dojin; Kumamoto, Japan) with a hematoxylin counterstain. Negative controls were run in parallel by replacing the specific antibody with $0.01 \mathrm{M}$ phosphate-buffered saline $(\mathrm{pH} 7.4)$ or mouse $\mathrm{IgG}$ (Dako; Glostrup, Denmark).

\subsection{Evaluation of the Expression of Biomarkers Using Stain Index}

Stain index, calculated as the product of tumor cell density and intensity of biomarker expression, was used for simple 
and comparable evaluation of the expression of biomarkers [23]. Tumor cell density per section was assessed by visual inspection and allocated scores of 1, 2, 3, 4, 5, and 6 for sparse, low, low/moderate, moderate, moderate/high, and high, respectively. Intensity of biomarker expression was estimated on a five-point scale $(0, \pm, 1+, 2+$, and $3+)$. If a homogeneous biomarker expression pattern was obtained, scores of $0, \pm, 1+, 2+$, and $3+$ were $0,1,2,4$, and 6 , respectively. In cases of a heterogeneous pattern, if predominant intensity is higher than minority intensity, the score is decreased by about $10 \%$ for each scale difference from the predominant staining intensity score. If minority intensity is higher than predominant intensity, the score is increased by about $20 \%$ for each scale difference from the predominant staining intensity score. Details are shown in Appendix A.

Stain index scores were evaluated by consultation between a pathologist and a radiation oncologist, who were blinded to all clinical information. The stain index scores of CD44, COX-2, and TWIST on the surface part (within the mucosal area or submucosal area) of the cancer and those of CD44, COX-2, and TWIST in the deep part (deeper than the submucosal area) of the cancer were different. Therefore, the surface part and deep part were evaluated separately for CD44, COX-2, and TWIST. Representative cases of biomarker expression are shown in Figure 1.

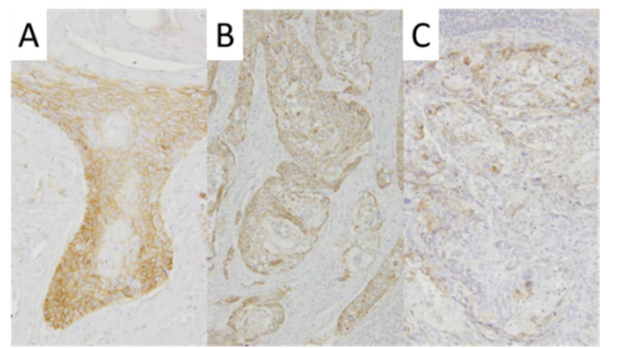

Figure 1. Representative cases of biomarker expression. (A) CD44 expression in the submucosa. Stain index was 6.0. (B) COX-2 expression in the proper muscle layer. Stain index was 4.0. (C) VEGF-C expression in the submucosa. Stain index was 0.3

\subsection{Study Endpoints}

The primary endpoint of this study was regional lymph node recurrence. Secondary endpoints were local control, distant metastasis, and OS.

\subsection{Statistical Analysis}

For this pilot study, we estimated duration to reginal failure of 6 months and estimated duration of OS in a regional control group of 42 months. When assuming a power of $80 \%$ and significance level of $5 \%$ then required sample-size was 12 . The follow-up time was calculated from the date when treatment started including the date of preoperative chemotherapy to the date of the last follow-up. Progression-free survival and OS were calculated using the Kaplan Meier method. Log-rank tests were used to compare regional control, distant control, and regional failure or pathological node-positive status within clinical node-negative status among subgroups based on patient-related and stain index score-related factors including age, CD44 of the cancer deep part, CD44 of the cancer surface part, COX-2 of the cancer deep part, COX-2 of the cancer surface part, TWIST of the cancer deep part, TWIST of the cancer surface part, LOX of the cancer, and VEGF-C of the cancer. These variables were divided into two groups by median values. Multivariate analysis was not performed due to the limited number of patients. P values $<0.05$ were considered statistically significant. All statistical analyses were performed using R software, version 3.4.4.

\section{Results}

\subsection{Baseline Characteristics}

The median age of the patients was 69 years (range: 59-82 years). The male-to-female ratio was $2.5(10 / 4)$. The most common primary tumor location was the lower segment of the esophagus ( 8 patients, $57.1 \%$ ) followed by the middle segment of the esophagus (5 patients, 35.7\%). Seven (50.0\%) of the 14 patients presented with locally advanced stage T3 cancer. Seven $(50.0 \%)$ of the patients received preoperative chemotherapy with cisplatin and 5-fluorouracil. The patients' baseline characteristics are shown in Table 1.

Table 1. Baseline Characteristics.

\begin{tabular}{|c|c|c|}
\hline Characteristics & & Number (\%) \\
\hline Number of patients & & $14(100.0)$ \\
\hline Sex & male/female & $10(71.4) / 4(28.6)$ \\
\hline Age & median/range, years & $69(59-82)$ \\
\hline Performance status & $0 / 1$ & $5(35.7) / 9(64.3)$ \\
\hline Histologic type & $\begin{array}{l}\text { Squamous cell } \\
\text { carcinoma }\end{array}$ & $14(100.0)$ \\
\hline \multirow{2}{*}{$\begin{array}{l}\text { Differentiation } \\
\text { Primary site in } \\
\text { esophagus }\end{array}$} & well/modelate/poor & $5(35.7) / 5(35.7) / 4(28.6)$ \\
\hline & $\begin{array}{l}\text { Cervico-upper/Middle/ } \\
\text { Lower }\end{array}$ & $1(7.1) / 5(35.7) / 8(57.1)$ \\
\hline \multirow{6}{*}{$\begin{array}{l}\text { Clinical TNM } \\
\text { staging }\end{array}$} & cT1N0M0 & $2(14.3)$ \\
\hline & cT1N1M0 & $2(14.3)$ \\
\hline & cT2N0M0 & $1(7.1)$ \\
\hline & cT3N0M0 & $2(14.3)$ \\
\hline & cT3N1M0 & $2(14.3)$ \\
\hline & cT3N2M0 & $3(21.4)$ \\
\hline \multirow{7}{*}{$\begin{array}{l}\text { Pathological TNM } \\
\text { staging }\end{array}$} & pT1N0M0 & $3(21.4)$ \\
\hline & pT1N1M0 & $1(7.1)$ \\
\hline & pT1N2M0 & $1(7.1)$ \\
\hline & pT2N0M0 & $2(14.3)$ \\
\hline & pT3N0M0 & $1(7.1)$ \\
\hline & pT3N1M0 & $3(21.4)$ \\
\hline & pT3N2M0 & $3(21.4)$ \\
\hline \multicolumn{3}{|l|}{ Surgery } \\
\hline Esophagectomy & subtotal/total & $13(92.9) / 1(7.1)$ \\
\hline Node dissection & 2 -field/3-field & $12(85.7) / 2(14.3)$ \\
\hline Chemotherapy & neoadjuvant & $7(50.0)$ \\
\hline
\end{tabular}

\subsection{Treatment Outcomes}

The median follow-up period was 38 months (range, 5-63 months). The actuarial overall survival (OS) rates at 18 and 36 months were $85.1 \%$ (95\% confidence interval [CI]: 52.3-96.1) and $69.6 \%$ (95\% CI: 37.8-87.4), respectively. The disease-free 
survival (DFS) rates at 18 and 36 months were 76.2\% (95\% CI: 42.7-91.7) and 59.2\% (95\% CI: 27.9-80.7), respectively. Regional control rates at 18 and 36 months were $84.6 \%(95 \%$ CI: 51.2-95.9) and 76.2\% (95\% CI: 42.7-91.7), respectively.

A

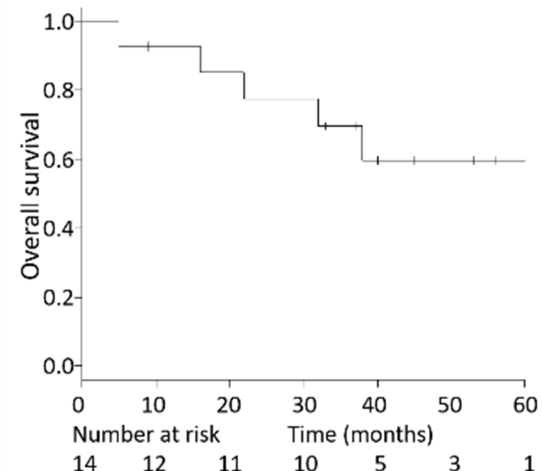

Distant control rates at 18 and 36 months were $83.9 \%$ (95\% CI: 49.4-95.7) and 67.1\% (95\% CI: 34.2-86.2), respectively. OS and DFS rates are shown in Figure 2.
B

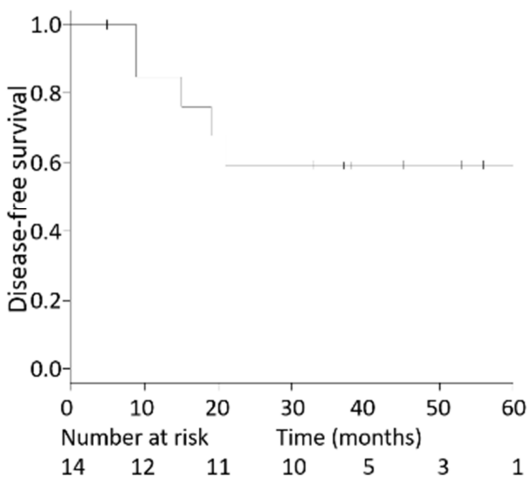

Figure 2. Kaplan Meier curves of overall survival and disease-free survival of the entire cohort. (A) Overall survival. (B) Disease-free survival.

Five patients had recurrence. The median time to overall recurrence was 15 months. Three of those 5 patients had regional recurrence and 4 patients had distant metastasis. Two of the 5 patients had regional recurrence and distant metastasis simultaneously. The median times to regional failure and distant metastasis were 9 months and 17.5 months, respectively. None of the patients had local recurrence.

\subsection{Analysis of Factors Affecting Regional Control and Distant Control}

Univariate analysis using the log-rank test was performed to evaluate associations of biomarkers expression between variables in the regional control group and regional failure group, between variables in the distant control group and distant failure group, and between variables of regional failure or pathological node-positive status within clinical node-negative status.

Results of univariate analysis showed that patients $\geq 69$ years of age had better regional control than did patients $<69$ years of age (3-year regional control rate: $100.0 \%$ vs $50.0 \%$; $\mathrm{p}=0.0444)$. Analysis of stain index scores showed that patients with CD44 of the cancer surface part $<3.0$ tended to have regional control, though there was no statistically significant association (3-year regional control rate: $100.0 \%$ vs $57.1 \% ; \mathrm{p}=0.0902)$. Patinets with COX-2 of the cancer deep part $\geq 2.8$ had better distant control (3-year distant control rate: $100.0 \%$ vs $42.9 \%$; $=0.0479)$. Patients with VEGF-C $<0.3$ had more regional failure or pathological node-positive status within clinical node-negative status (3-year failure rate: $53.5 \%$ vs $100.0 \%$; $\mathrm{p}=0.0418$ ). Comparisons of different variables are shown in Table 2.

Table 2. Univariate Analysis of Stain Indices of Biomarkers.

\begin{tabular}{|c|c|c|c|c|c|c|c|c|}
\hline $\begin{array}{l}\text { Prognostic } \\
\text { variables }\end{array}$ & Category & No. of patients & $\begin{array}{l}\text { 3-year regional } \\
\text { control }(\%)\end{array}$ & $p$ value & $\begin{array}{l}\text { 3-year distant } \\
\text { control }(\%)\end{array}$ & $p$ value & $\begin{array}{l}\text { 3-year } \geq \mathrm{pN} 1 \text { or regional } \\
\text { failure in } \mathrm{cN0}(\%)\end{array}$ & p value \\
\hline \multirow{2}{*}{ Age } & $<69$ & 6 & 50.0 & \multirow{2}{*}{0.0444} & 50.0 & \multirow{2}{*}{0.168} & 66.7 & \multirow{2}{*}{0.124} \\
\hline & $\geq 69$ & 8 & 100.0 & & 83.3 & & 100.0 & \\
\hline \multirow{2}{*}{ Clinical T stage } & cT1 & 4 & 75.0 & \multirow{2}{*}{0.913} & 50.0 & \multirow{2}{*}{0.248} & 75.0 & \multirow{2}{*}{0.513} \\
\hline & $\geq \mathrm{cT} 2$ & 10 & 76.2 & & 75.0 & & 87.5 & \\
\hline \multirow{2}{*}{ CD44 deep } & $<2.0$ & 5 & 75.0 & \multirow{2}{*}{0.837} & 50.0 & \multirow{2}{*}{0.591} & 75.0 & \multirow{2}{*}{0.713} \\
\hline & $\geq 2.0$ & 9 & 75.0 & & 75.0 & & 87.5 & \\
\hline \multirow{2}{*}{ CD44 surface } & $<3.0$ & 6 & 100.0 & \multirow{2}{*}{0.0902} & 80.0 & \multirow{2}{*}{0.330} & 100.0 & \multirow{2}{*}{0.194} \\
\hline & $\geq 3.0$ & 8 & 57.1 & & 57.1 & & 71.4 & \\
\hline \multirow{2}{*}{ COX-2 deep } & $<2.8$ & 7 & 71.4 & \multirow{2}{*}{0.698} & 42.9 & \multirow{2}{*}{0.0479} & 71.4 & \multirow{2}{*}{0.194} \\
\hline & $\geq 2.8$ & 7 & 83.3 & & 100.0 & & 100.0 & \\
\hline \multirow{2}{*}{ COX-2 surface } & $<3.8$ & 7 & 71.4 & \multirow{2}{*}{0.698} & 57.1 & \multirow{2}{*}{0.330} & 71.4 & \multirow{2}{*}{0.194} \\
\hline & $\geq 3.8$ & 7 & 83.3 & & 80.0 & & 100.0 & \\
\hline \multirow{2}{*}{ TWIST deep } & $<0.0$ & 10 & 66.7 & \multirow{2}{*}{0.238} & 66.7 & \multirow{2}{*}{0.998} & 77.8 & \multirow{2}{*}{0.365} \\
\hline & $\geq 0.0$ & 4 & 100.0 & & 66.7 & & 100.0 & \\
\hline \multirow{2}{*}{ TWIST surface } & $<0.6$ & 7 & 71.4 & \multirow{2}{*}{0.698} & 57.1 & \multirow{2}{*}{0.443} & 71.4 & \multirow{2}{*}{0.194} \\
\hline & $\geq 0.6$ & 7 & 83.3 & & 80.0 & & 100.0 & \\
\hline \multirow{2}{*}{ LOX } & $<0.3$ & 6 & 60.0 & \multirow{2}{*}{0.246} & 80.0 & & 80.0 & \\
\hline & $\geq 0.3$ & 8 & 85.7 & & 57.1 & 0.544 & 85.7 & 0.713 \\
\hline VFGF-C & $<0.3$ & 6 & 53.3 & 0246 & 53.3 & 0364 & 53.3 & 00418 \\
\hline VEGF-C & $\geq 0.3$ & 8 & 87.5 & 0.246 & 75.0 & 0.364 & 100.0 & 0.0418 \\
\hline
\end{tabular}




\section{Discussion}

In this study, we evaluated relationships of the expression levels of biomarkers with 1) distant failure, 2) regional control, and 3) $\geq \mathrm{pN} 1$ or regional failure in cases of $\mathrm{cN} 0$. These comparisons were made because 1) reduction of the prophylactic radiation field and delivery of chemotherapy might be important in cases of distant failure, 2) reduction of the prophylactic radiation field might be possible in cases of regional control, and 3) reduction of the prophylactic radiation field might be a risk for regional failure in cases of $\geq \mathrm{pN} 1$ or regional failure within $\mathrm{cN} 0$.

Possible strategy for shrinking or omitting the prophylactic radiation field is prediction of distant failure. In cases of distant failure, control of distant failure by systemic chemotherapy might be more important than regional control by prophylactic radiation. Furthermore, prophylactic radiation to a large field might cause lung and heart toxicities and might make the acceptance of chemotherapy difficult. If distant failure is predictable in cases of radiotherapy for preoperative chemotherapy failure and in cases of postoperative radiotherapy for surgical incomplete resection, it might be possible to reduce the prophylactic radiation field in such cases and thus to decrease the risk of radiation-induced toxicities and decrease the influence on acceptance of chemotherapy.

In this study, patients with a low level of COX-2 expression in the deep part of the cancer (stain index $<2.8$ ) had significantly worse distant control (3-year distal control rate: $100.0 \%$ vs $42.9 \% ; \mathrm{p}=0.0479$ ). $\mathrm{COX}-2$ is known to enhance tumor cell invasiveness, stimulation of neovascularization, and immunosuppression [24]. Therefore, we expected that patients with a higher level of COX-2 expression in the deep part of the cancer world show significantly worse distant control, as $\mathrm{Hu}$ et al. [25] reported. However, we obtained completely opposite results. We assumed that the opposite results were due to preoperative chemotherapy. Sivula et al. [26] reported that a high level of COX-2 expression was associated with distant metastasis in patients who did not receive neoadjuvant chemotherapy, whereas a low level of COX-2 expression was associated with distant metastasis in patients who received neoadjuvant chemotherapy. Furthermore, a low level of COX-2 expression was a significant prognostic factor (relative risk: 7.5). In the present study, 7 (50.0\%) of the patients received preoperative chemotherapy. This could influence the results. From these findings, a low level of COX-2 expression in the deep part of the cancer (stain index $<2.9$ ) might be a biomarker that enables shrinkage or omission of the prophylactic radiation field in cases of radiotherapy for preoperative chemotherapy failure or postoperative radiotherapy for surgical incomplete resection.

In this study, patients with a low level of VEGF-C expression (stain index $<0.3$ ) had a significantly higher rate of regional failure or pathological node-positive status within clinical node-negative status (3-year failure rate: $53.5 \%$ vs $100.0 \%$; $=0.0418$ ). This implies that even in $\mathrm{cN} 0$ cases, a low level of VEGF-C expression could mean a hidden risk of lymph node metastasis. VEGF-C expression is known as a major inducer of angiogenesis, vessel permeability, lymphatic involvement, venous involvement, and lymph node metastasis [17-19]. Therefore, we expected that patients with a high level of VEGF-C expression would show a significantly worse pathological node-positive status or reginal failure. However, we obtained completely opposite results. We assumed that the opposite results were due to the clinical node-negative status. Kitadai et al. [19] and Pan et al. [27] reported that a high level of VEGF-C expression is related to clinical node-positive stasus and that a low level of VEGF-C expression is related to clinical node-negative status. Though, the present study implies that a low level of VEGF-C expression in the cancer does not necessarily mean pN0 and regional control in cases of cN0. Further study is required to determine how to deal with VEGF-C expression.

Another possible strategy for reduction of the prophylactic radiation field might prediction of regional control. The results of this study showed that patients with lower CD44 expression in the cancer surface part (stain index <3.0) tended to have better regional control, though the difference was not statistically significant (3-year regional control rate: $100.0 \%$ vs $57.1 \%$; $\mathrm{p}=0.0902$ ). CD44 is known as an adhesive molecule that can connect to the extracellular matrix by intercellular adhesion or cell-matrix adhesion [28]. CD44 is also related to epithelial-mesenchymal transition, cell movement, and metastasis [29]. Therefore, upregulation of CD44 expression on the cancer surface might represent acquisition of invasive capacity and lymph node metastasis capacity. Liu et al. [30] reported that a high CD44 expression level is a prognostic factor of local failure-free survival (hazard ratio: 1.75$)$. The present study included results from $\geq \mathrm{cN} 1$ patients who received 2-3-field lymph node dissection. Therefore, simply applying these results to definitive chemoradiotherapy might be inappropriate. However, patients who had more advanced pathological node staging than clinical node staging showed high CD44 expression. From these findings, a low level of CD44 expression in the cancer surface might be a biomarker that enables shrinkage or omission of the prophylactic radiation field.

Other than biomarker expression, patients $\geq 69$ years of age had significantly better regional control than did patients $<69$ years of age (3-year regional control rate: $100.0 \%$ vs $50.0 \%$; $\mathrm{p}=0.0444$ ). We could not find any report in which advanced age was shown to be a factor of regional control. There was no difference in neoadjuvant chemotherapy received ratio between these two groups. However, the possibility of selection bias cannot be completely ruled out.

Although this study was the first study that was carried out to try to determine cases in which the prophylactic radiation field can be reduced or omitted based on expression levels of 
biomarkers in tumors using a quantitative stain index, it has several limitations. It was a retrospective analysis with a small sample size. The two subsets of patients could not balance well because of the study's retrospective nature. The possibility of an influence of preoperative chemotherapy could not be excluded. Additionally, the results were obtained from surgically resected samples. For application of the results to chemoradiotherapy, a study using endoscopic biopsy samples may be required. However, obtaining endoscopic samples from the deep part of the cancer would be a challenge. For the next analysis, a well-balanced prospective registry study using CD44, COX-2, and VEGF-C expression in endoscopic biopsy samples is required before application to shrinkage or omittance of the prophylactic radiation field in a clinical trial.

\section{Conclusions}

Low CD44 expression or low COX-2 expression of the cancer might be a biomarker that enables safe shrinkage or omission of the prophylactic radiation field. In cases of radiotherapy for preoperative chemotherapy failure or postoperative radiotherapy for surgical incomplete resection, low COX-2 expression might indicate the possibility of shrinkage or omission of the prophylactic radiation field. A prospective registry study using endoscopic biopsy samples is required for application of these biomarkers to radical chemoradiotherapy.

\section{Appendix}

Table 3. Stain index scores used to quantitate expression of biomarkers.

\begin{tabular}{|c|c|}
\hline \multicolumn{2}{|l|}{ Tumor cell density score } \\
\hline $\begin{array}{r}\text { Proportion of cells } \\
\end{array}$ & Score \\
\hline sparse & 1 \\
\hline low & 2 \\
\hline low/moderate & 3 \\
\hline moderate & 4 \\
\hline moderate/high & 5 \\
\hline high & 6 \\
\hline
\end{tabular}

\begin{tabular}{|c|c|c|c|c|c|c|}
\hline \multicolumn{7}{|c|}{ Biomarker expression intensity score } \\
\hline \multicolumn{7}{|c|}{ Predominant intensity score } \\
\hline & & $\mathbf{0}$ & \pm & $1+$ & $2+$ & $3+$ \\
\hline \multirow{5}{*}{$\begin{array}{l}\text { Minority } \\
\text { intensity } \\
\text { score }\end{array}$} & 0 & 0.0 & 0.8 & 1.5 & 2.5 & 4.0 \\
\hline & \pm & 0.3 & 1.0 & 1.8 & 3.0 & 4.3 \\
\hline & $1+$ & 0.8 & 1.3 & 2.0 & 3.5 & 5.0 \\
\hline & $2+$ & 1.0 & 1.7 & 2.3 & 4.0 & 5.5 \\
\hline & $3+$ & 1.5 & 2.0 & 3.0 & 4.5 & 6.0 \\
\hline
\end{tabular}

\section{Funding}

This research was supported by Japan Society for the Promotion of Science (JSPS) KAKENHI Grant Number JP23951831. The funders had no role in the current study other than providing financial support.

\section{Conflicts of Interest}

All the authors do not have any possible conflicts of interest.

\section{Acknowledgements}

We would like to thank SES translation proofreading service for English language editing.

\section{References}

[1] Herskovic A, LeichmanL, Lattin P, Han I, Ahmad K, Leichman G, et al. Chemo/radiation with and without surgery in the thoracic esophagus: the Wayne State experience. Int J Radiat Oncol Biol Phys. 1988; 15 (3): 655-662 [Pubmed: 3138217] [doi: 10.1016/0360-3016(88)90308-2].

[2] Konski A, Doss M, Milestone B, Haluszka O, Hanlon A, Freedman G, et al. The integration of 18-fluoro-deoxy-glucose positron emission tomography and endoscopic ultrasound in the treatment-planning process for esophageal carcinoma. Int J Radiat Oncol Biol Phys. 2005; 61 (4): 1123-1128 [Pubmed: 15752892] [doi: 10.1016/j.ijrobp.2004.07.717].

[3] Toya R, Matsuyama T, Saito T, Imuta M, Shiraishi S, Fukugawa Y, et al. Impact of hybrid FDG-PET/CT on gross tumor volume definition of cervical esophageal cancer: reducing interobserver variation. J Radiat Res. 2019; 60 (3): 348-352 [Pubmed: 30864652] [doi: 10.1093/jrr/rrz004].

[4] Schröder W, Baldus SE, Mönig SP, Beckurts TK, Dienes HP, Hölscher AH. Lymph node staging of esophageal squamous cell carcinoma in patients with and without neoadjuvant radiochemotherapy: histomorphologic analysis. World J Surg. 2002; 26 (5): 584-587 [Pubmed: 12098049] [doi: 10.1007/s00268-001-0271-5].

[5] Chang EY, Li X, Jerosch-Herold M, Priest RA, Enestvedt CK, $\mathrm{Xu} \mathrm{J}$, et al. The evaluation of esophageal adenocarcinoma using dynamic contrast-enhanced magnetic resonance imaging. J Gastrointest Surg. 2008; 12 (1): 166-175 [Pubmed: 17768665] [doi: 10.1007/s11605-007-0253-5].

[6] Minsky BD, Pajak TF, Ginsberg RJ, Pisansky TM, Martenson J, Komaki R, et al. INT 0123 (Radiation Therapy Oncology Group 94-05) phase III trial of combined-modality therapy for esophageal cancer: high-dose versus standard-dose radiation therapy. J Clin Oncol. 2002; 20 (5): 1167-1174 [Pubmed: 11870157] [doi: 10.1200/JCO.2002.20.5.1167].

[7] Kato K, Muro K, Minashi K, Ohtsu A, Ishikura S, Boku N, et al. Phase II study of chemoradiotherapy with 5-fluorouracil and cisplatin for stage II-III esophageal squamous cell carcinoma: JCOG trial (JCOG 9906). Int J Radiat Oncol Biol Phys. 2011; 81 (3): 684-690 [Pubmed: 20932658] [doi: 10.1016/j.ijrobp.2010.06.033].

[8] Kato H, Sato A, Fukuda H, Kagami Y, Udagawa H, Togo A, et al. A phase II trial of chemoradiotherapy for stage I esophageal squamous cell carcinoma: Japan Clinical Oncology Group Study (JCOG 9708). Jpn J Clin Oncol. 2009; 39 (10): 638-643 [Pubmed: 19549720] [doi: 10.1093/jjco/hyp069].

[9] Collard JM, Otte JB, Fiasse R, Laterre PF, De Kock M, Longueville J, et al. Skeletonizing en bloc esophagectomy for cancer. Ann Surg. 2001; 234 (1): 25-32 [Pubmed: 11420480] [doi: 10.1097/00000658-200107000-00005]. 
[10] Dresner SM, Lamb PJ, Bennett MK, Hayes N, Griffin SM. The pattern of metastatic lymph node dissemination from adenocarcinoma of the esophagogastric junction. Surgery. 2001; 129 (1): 103-109 [Pubmed: 11150040] [doi: 10.1067/msy.2001.110024].

[11] Akiyama H, Tsurumaru M, Kawamura T, Ono Y. Principles of surgical treatment for carcinoma of the esophagus: analysis of lymph node involvement. Ann Surg. 1981; 194 (4): 438-446 [Pubmed: 7283505] 10.1097/00000658-198110000-00007].

[12] Huang W, Li B, Gong H, Yu J, Sun H, Zhou T, et al. Pattern of lymph node metastases and its implication in radiotherapeutic clinical target volume in patients with thoracic esophageal squamous cell carcinoma: a report of 1077 cases. Radiother Oncol. 2010; 95 (2): 229-233 [Pubmed: 20189259] [doi: 10.1016/j.radonc.2010.01.006].

[13] Ishikura S, Nihei K, Ohtsu A, Boku N, Hironaka S, Mera K, et al. Long-term toxicity after definitive chemoradiotherapy for squamous cell carcinoma of the thoracic esophagus. J Clin Oncol. 2003; 21 (14): 2697-2702 [Pubmed: 12860946] [doi: 10.1200/JCO.2003.03.055].

[14] Morota M, Gomi K, Kozuka T, Chin K, Matsuura M, Oguchi $\mathrm{M}$, et al. Late toxicity after definitive concurrent chemoradiotherapy for thoracic esophageal carcinoma. Int $\mathrm{J}$ Radiat Oncol Biol Phys. 2009; 75 (1): 122-128 [Pubmed: 19327900] [doi: 10.1016/j.ijrobp.2008.10.075].

[15] Gotoda T, Matsumura Y, Kondo H, Ono H, Kanamoto A, Kato $\mathrm{H}$, et al. Expression of CD44 variants and prognosis in oesophageal squamous cell carcinoma. Gut. 2000; 46 (1): 14-19 [Pubmed: 10601048] [doi: 10.1136/gut.46.1.14].

[16] Huang JX, Chen WC, Lin M, Zhang YL, Li FY, Song ZX, et al. Clinicophathological significance of cyclooxygenase-2 and cell cycle-regulatory proteins expression in patients with esophageal squamous cell carcinoma. Dis Esophagus. 2012; 25 (2): 121-129 [Pubmed: 21762277] [doi: 10.1111/j.1442-2050.2011.01219.x].

[17] Byeon JS, Jung HY, Lee YJ, Lee D, Lee GH, Myung SJ, et al. Clinicophathological significance of vascular endothelial growth factor-C and cyclooxygenase- 2 in esophageal squamous cell carcinoma. J Gastroenterol Hepatol. 2004; 19 (6): 648-654 [Pubmed: 15151619] [doi: 10.1111/j.1440-1746.2004.03348.x].

[18] Han U, Can OI, Han S, Kayhan B, Onal BU. Expressions of p53, VEGF C, p21: could they be used in preoperative evaluation of lymph node metastasis of esophageal squamous cell carcinoma? Dis Esophagus. 2007; 20 (5): 379-385 [Pubmed: 17760650] [doi: 10.1111/j.1442-2050.2007.00634.x].

[19] Kitadai Y, Amioka T, Haruma K, Tanaka S, Yoshihara M, Sumii K, et al. Clinicopathological significance of vascular endothelial growth factor (VEGF)-C in human esophageal squamous cell carcinomas. Int J Cancer. 2001; 93 (5): 662-666 [Pubmed: 11477575] [doi: 10.1002/ijc.1379].

[20] Sakai M, Kato H, Sano A, Tanaka N, Inose T, Kimura H, et al. Expression of lysyl oxidase is correlated with lymph node metastasis and poor prognosis in esophageal squamous cell carcinoma. Ann Surg Oncol. 2009; 16 (9): 2494-2501 [Pubmed: 19526206] [doi: 10.1245/s10434-009-0559-5].

[21] Donahue JM, Nichols FC, Li Z, Schomas DA, Allen MS, Cassivi SD, et al. Complete pathologic response after neoadjuvant chemoradiotherapy for esophageal cancer is associated with enhanced survival. Ann Thorac Surg. 2009; 87 (2): 392-398 [Pubmed: 19161745] [doi: 10.1016/j.athoracsur.2008.11.001].

[22] Ando N, Kato H, Igaki H, Shinoda M, Ozawa S, Shimizu H, et al. A randomized trial comparing postoperative adjuvant chemotherapy with cisplatin and 5-fluorouracil versus preoperative chemotherapy for localized advanced squamous cell carcinoma of the thoracic esophagus (JCOG9907). Ann Surg Oncol. 2012; 19 (1): 68-74 [Pubmed: 21879261] [doi: 10.1245/s10434-011-2049-9].

[23] King RJ, Coffer AI, Gilbert J, Lewis K, Nash R, Millis R, et al. Histochemical studies with a monoclonal antibody raised against a partially purified soluble estradiol receptor preparation from human myometrium. Cancer Res. 1985; 45 (11pt2): 5728-5733 [Pubmed: 2413995].

[24] Rizzo MT. Cyclooxygenase-2 in oncogenesis. Clin Chim Acta. 2011; 412 (9-10): 671-687 [Pubmed: 21187081] [doi: 10.1016/j.cca.2010.12.026].

[25] Hu Z, Yang Y, Zhao Y, Huang Y. The prognostic value of cyclooxygenase-2 expression in patients with esophageal cancer: evidence from a meta-analysis. Onco Targets Ther. 2017; 10: 2893-2901 [Pubmed: 28652771] [doi: 10.2147/OTT.S134599].

[26] Sivula A, Buskens CJ, van Rees BP, Haglund C, Offerhaus GJ, van Lanschot JJ, et al. Prognostic role of cyclooxygenase-2 in neoadjuvant-treated patients with squamous cell carcinoma of the esophagus. Int J Cancer. 2005; 116 (6): 903-908 [Pubmed: 15856454] [doi: 10.1002/ijc.21134].

[27] Pan X, Mao T, Fang W, Chen W. Vascular endothelial growth factor $\mathrm{C}$ is an indicator of lymph node metastasis in thoracic esophageal squamous cell carcinomas and its role in long-term survival after surgery. Thrac Cancer. 2014; 5 (4): 313-318 [Pubmed: 26767018] [doi: 10.1111/1759-7714.12094].

[28] Nair KS, Naidoo R, Chetty R. Expression of cell adhesion molecules in oesophageal carcinoma and its prognostic value. J Clin Pathol. 2005; 58 (4): 343-351 [Pubmed: 15790695] [doi: $10.1136 /$ jcp.2004.018036].

[29] Kinugasa H, Whelan KA, Tanaka K, Natsuizaka M, Long A, Guo A, et al. Mitochondrial SOD2 regulates epithelial-mesenchymal transition and cell populations defined by differential CD44 expression. Oncogene. 2015; 34 (41): 5229-5239 [Pubmed: 25659582] [doi: 10.1038/onc.2014.449].

[30] Liu S, Anfossi S, Qiu B, Zheng Y, Cai M, Fu J, et al. Prognostic factors for locoregional recurrence in patients with thoracic esophageal squamous cell carcinoma treated with radical two-field lymph node dissection: results from long-term follow-up. Ann Surg Oncol. 2017; 24 (4): 966-973 [Pubmed: 27804027] [doi: 10.1245/s10434-016-5652-y]. 